ORIGINAL RESEARCH PAPER

\section{IMPACT OF VACCINATION ON COVID INFECTION IN HEALTH CARE WORKERS IN WESTERN ZONE OF INDIAN RAILWAYS}

\section{Epidemiology}

KEY WORDS: Post vaccination, Healthcare workers, COVID-19 infection, Delta plus variant, Prevalence, Disease severity

\begin{tabular}{ll}
\hline Dr. C.S. Anand & Principal Chief Medical Director,Western Railway \\
\hline $\begin{array}{l}\text { Dr. Hafeezunissa } \\
\text { Rehman }\end{array}$ & Medical Director,Jagjivanram Hospital Western Railways \\
\hline $\begin{array}{l}\text { Dr. K. Krishna } \\
\text { Kumar }\end{array}$ & CMS-Vadodara \\
$\begin{array}{l}\text { Dr. Brham } \\
\text { Prakash }\end{array}$ & CMS-Dahod \\
\hline $\begin{array}{l}\text { Dr. Subodh } \\
\text { Kumar }\end{array}$ & CMS-Bhavnagar \\
\hline $\begin{array}{l}\text { Dr. A. K. Malviya } \\
\text { Dr. Maniratan } \\
\text { Chakraborty }\end{array}$ & CMS-Rajkot \\
\hline $\begin{array}{l}\text { Dr. Alok } \\
\text { Srivastava }\end{array}$ & CMS-Ahemadabad \\
\hline $\begin{array}{l}\text { Dr. J.P. Rawat } \\
\begin{array}{l}\text { Dr Yoganand } \\
\text { Patil }\end{array}\end{array}$ & CMS-Mumbai division \\
\hline $\begin{array}{l}\text { Dr Mrunal } \\
\text { Kesari* }\end{array}$ & MospitalWestern Railways \\
\hline
\end{tabular}

\begin{tabular}{l|l} 
Healthcare workers (HCWs) are highly exposed to SARS-CoV-2 infection. HCWs are also likely source of infection of \\
their family members .COVID-19 Vaccination programs started during December 2020 in several countries and \\
prioritized healthcare workers (HCWs). The vaccination campaign coincided with the second surge of COVID cases in
\end{tabular}

INTRODUCTION:

The novel coronavirus 2019 (COVID-19) continues to be a significant health issue worldwide. Infection caused by severe acute respiratory syndrome coronavirus-2 (SARS CoV2) may be associated with a wide range of disease patterns ranging from mild to life threatening pneumonia and multiorgan dysfunction syndrome.[1] It was declared a pandemic by WHO on March 10, 2020 [2]. Health-care workers (HCWs) are Amongst the highest population at the risk of exposure to the disease.[3]

Several variants of the SARS CoV-2 have been reported in India during January to April 202 1: (SARSCoV-2- B. 1.1.7 (alpha variant, United Kingdom), B.1.351(beta variant, South Africa), B.1.617.2 (delta) variant, B.1.1.28 (Brazil P1, P2)].[4,5] The B.1.617.2 (delta) variant of the SARS-CoV-2, was detected across the globe and most of which have been reported from India, starting in mid-April 2021 and a notable increase in cases seen in the United Kingdom.[6] The delta variant is characterized by the mutations in the spike protein T19R, $\Delta 157-158$, L452R, T478K, D614G, P681R, and D950N. These mutations may affect immune responses and it appears that mutations at these sites may have increased replication, which leads to higher viral loads and increased transmission of these strains.[7,8] The Delta Plus variant - also known as B.1.617.2.1 or AY.1 - is different from delta variant because it contains a new mutation in the spike protein-K4 17N. Delta Plus variant have three characteristics: increased transmissibility, stronger binding to receptors of lung cells (suggesting easier entry into human cells), and a potential reduction in response to monoclonal antibodies (so that they may be less responsive to lifesaving monoclonal antibody treatments). [9]

COVID-19 Vaccination programs started during December 2020 in several countries and prioritized healthcare workers (HCWs).[10] Two vaccines that have been granted emergency use authorization by the Central Drugs Standard Control Organization (CDSCO) in India are Covishield (AstraZeneca's vaccine manufactured by Serum Institute of India) and Covaxin (manufactured by Bharat Biotech Limited).[11] Recently the Indian government granted emergency use approval (EUA) to US-based pharma company Moderna vaccine and SputnikV created by Gamaleya Institute in Moscow.[12] The vaccination campaign 
coincided with a second surge of COVID cases.Surveillance is one of the significant phases in controlling viral diseases, which can be achieved either by active or passive approaches.

Western railway covers most parts of Gujrat, western parts of Madhya Pradesh and Maharashtra. The Medical department of western railway provides comprehensive health care i.e. Administrative, Curative, Preventive and Promotive services. These services are provided at three levels i.e. Health units (Primary Health Care- basic Health Care), Sub divisional, Divisional Hospitals, Workshop Hospital (Secondary Health Care- speciality health care) and Zonal Hospital (Tertiary Health Care- speciality\& super speciality health care). The beneficiaries include serving railway employees and their family members and dependents, retired railway employees, their family members and dependents and certain other categories of staff such as contractors, labour, vendors, licensed porters etc. numbering 5.5 Lakhs.

\section{Aims:}

1. To assess the prevalence of COVID19 infection in vaccinated HCWs

2. To study post vaccination disease severity in HCWs.

3. To study coverage of COVID 19 vaccination program by western railway.

\section{Material and Methods:}

This is a retrospective cross sectional cohort study conducted in Health Care workers of Western Zone of Indian Railways. INDIA launched a vaccination campaign in early January 2021, and HCWs of the study center received Covishield vaccine. SARS-CoV 2 cases in HCWs were detected by real time reverse transcriptase polymerase chain reaction (rRTPCR) and rapid antigen test (RAT). Testing was done for symptomatic cases, asymptomatic HCWs for workplace exposure as pat of contact tracing, out state travel and as per request. Vaccinated HCWs who were infected with COVID from February till June 2021 are included in this study. A telephonic survey was done to collect data about dates of vaccination of first and second dose, Date of positive report, symptoms, severity and hospitalization.

\section{RESULT:}

Out of total 2314 HCWs of Western Railway, 2140 (92.48\%) HCWs were vaccinated from February till June 2021 (Chart 1). Full dose of vaccination was completed by $38.2 \%(817 / 2140)$ HCWs while $61.8 \%(1323 / 2140)$ had taken only one dose (Chart 2). Total HCWs infected with COVID-19 after vaccination was 135 . Of the total $2314 \mathrm{HCWs}, 174$ had not received vaccination. Prevalence of SARS-CoV-2 infection amongst vaccinated $\mathrm{HCW}$ was $6.3 \%$. Percentage of infection in HCW who took a single dose was $7.7 \%$ and $4 \%$ in cases receiving both vaccine doses (Table 1, Chart 2).Cases occurring within 15 days from initial dose of vaccination were $60.7 \%(82 / 135)$ and $39.3 \%(53 / 135)$ cases occurred after 15 days of vaccination (Table 2, Chart 3 ). Cases managed at home isolation were $82.9 \%$ and $17.1 \%$ were admitted in hospital for treatment (Table 3, Chart 4). $87.4 \%$ of the infected HCWs had Mild disease, $11.1 \%$ had moderate disease. There were two severe cases $(1.5 \%)$, out of which one succumbed to infection (Table 4, Chart 5).

Table 1: Percentage (\%) of infected HCWafter receiving vaccination

\begin{tabular}{|c|c|c|c|}
\hline Vaccine dose & $\begin{array}{c}\text { Total HCW } \\
\text { receiving }\end{array}$ & $\begin{array}{c}\text { Positive } \\
\text { cases after }\end{array}$ & $\begin{array}{c}\text { Percentage (\%) } \\
\text { of infected HCW } \\
\text { after }\end{array}$ \\
\hline lst dose & 1323 & 102 & $7.7 \%$ \\
\hline 2nd dose & 817 & 33 & $4 \%$ \\
\hline Not vaccinated & 174 & & \\
\hline
\end{tabular}

Table 2: HCWs Characteristics Stratified by Vaccinationto-Diagnosis Timing

\begin{tabular}{|c|c|}
\hline <15 days of vaccination & $>$ 15days of vaccination \\
\hline $60.7 \%(82 / 135)$ & $39.3 \%(53 / 135)$ \\
\hline 32 & \\
\hline
\end{tabular}

Table 3: HCWs requiring home isolation and in hospital treatment

\begin{tabular}{|c|c|c|}
\hline Home isolation & 112 & $82.9 \%$ \\
\hline Hospitalization & 23 & $17.1 \%$ \\
\hline Total & 135 & $100 \%$ \\
\hline
\end{tabular}

Table 4: Categorization of HCWs according to disease severity

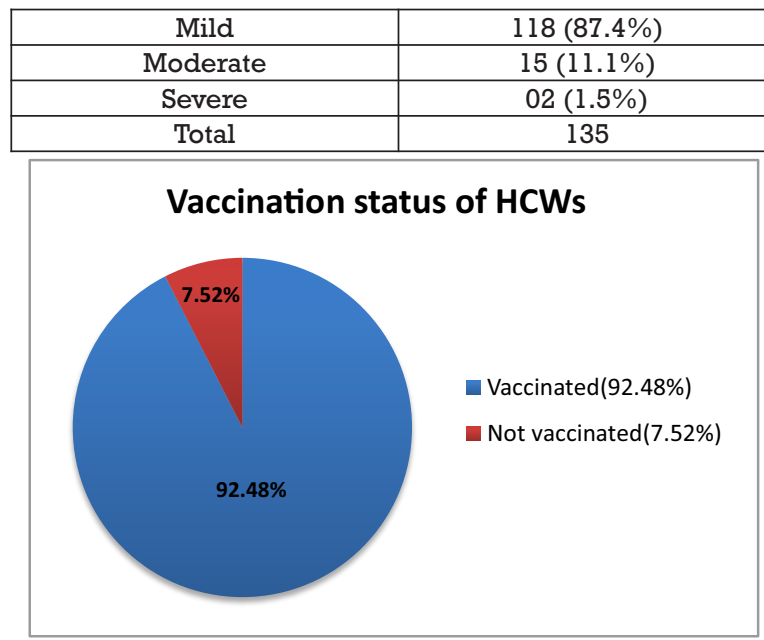

\section{Chart 1:Vaccination status of HCWs}

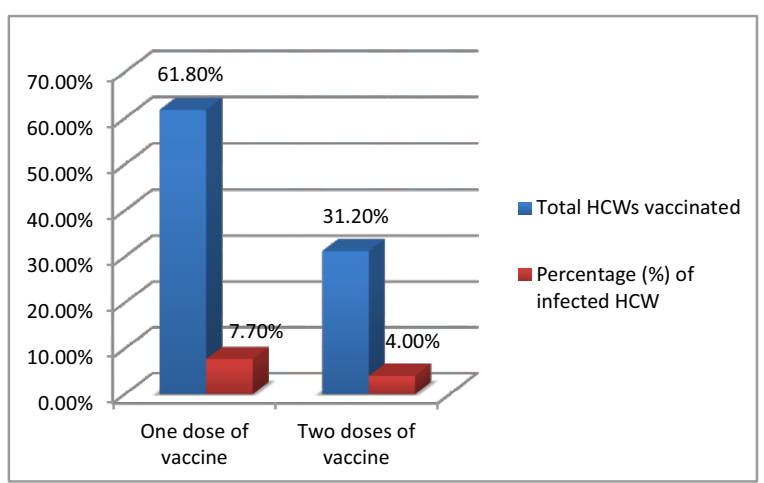

Chart 2: Percentage (\%) of infected HCW after receiving vaccination

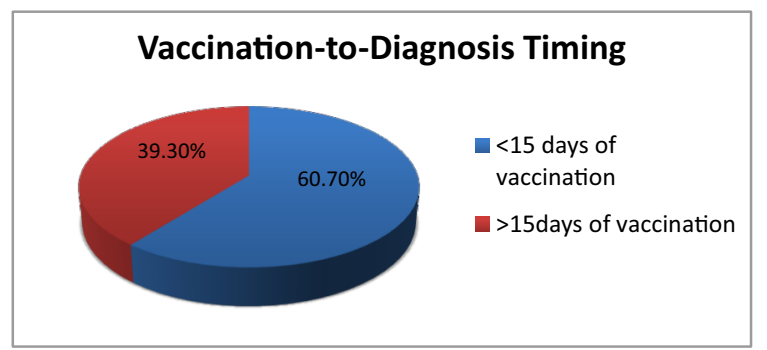

Chart 3: HCWs Characteristics Stratified by Vaccinationto-Diagnosis Timing

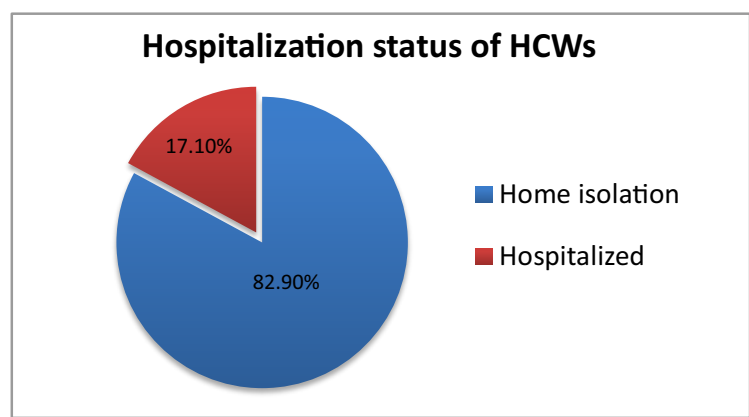

Chart 4: HCWs requiring home isolation and in hospital treatment 


\section{Disease severity among HCWs}

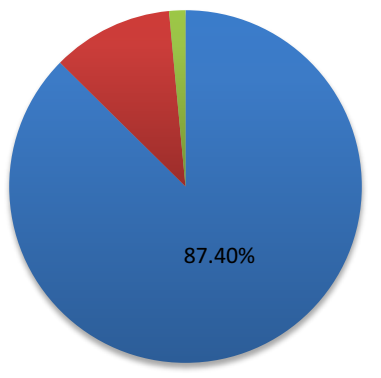

MILD (87.4\%)

- MODERATE(11.1\%)

- SEVERE(1.5\%)

Chart 5: Categorization of HCWs according to disease severity

\section{DISCUSSION:}

Covid pandemic has adversely affected the health and economy globally. India has experienced a high rise in cases of Covid-19 since late March 2021, reaching more than 400,000 cases and approximately 4000 deaths reported each day in early May 2021.[6,13] SARS-CoV-2 is different from other known viruses due to multiple mutations on the sites of nonstructural proteins (NSP) 2 and 3, and the varying nature of virulence between different persons.[14] WHO has been tracking mutations and variants since the start of the COVID19 outbreak. The global SARS-CoV-2 laboratory network includes a dedicated Virus Evolution Working Group, to detect new changes quickly and assess their possible impact. WHO has also established a SARS-CoV-2 Risk Monitoring and Evaluation Framework to identify, monitor and assess Covid variants of concern. It involves components like surveillance, research on variants of concern, and evaluation of the impact on diagnostics, therapeutics and vaccines. [15]

COVID-19 vaccination programs started during December 2020 in several countries, prioritizing higher risk groups including HCWs [10]Multiple mutant strains create challenges in the development of a universal vaccine. Designing a theranostic vaccine instead of a single shot to prevent multiple strains. Multiple vaccines are being developed globally. Different types of vaccine are: Live attenuated vaccines, inactivated vaccines, subunit vaccines, viral vector-based vaccines, and nucleic acid vaccines. Vaccine development is an extensive process with high chances of failure and even involves numerous challenges and safety issues to get accepted and approved.[14] Vaccines which are designed to elicit an IgG response, preventing the developing of COVID-19. However a local mucosal secretory IgA response as shown for anti-polio vaccination is required for protection against viral replication within the airways. Hence limited protection is offered by these vaccine[16,17] In India Covishield, Covaxin, Moderna and Sputnik V are the vaccines that have been granted emergency use authorization by the Central Drugs Standard Control Organization (CDSCO).[18]. It takes 2-3 weeks for adequate immune response after completion of the entire vaccination schedule.[11]

Post-vaccination breakthrough infection is defined as the contraction of a virus after being inoculated with a single or both doses of the vaccine. Post surveillance helps in identifying any unexpected adverse risk such as anaphylaxis, vaccine-strain systemic infection and vaccine-associated paralytic condition.[19] The first real-world case of positive PCR test in a HCW after full dose vaccination-induced immunity was reported in January in the United Kingdom in HCW after the Pfizer-BioNTech vaccine.[20] In a study done at hospital in Israel by Amit $\mathrm{S}$ et al, active and passive surveillance of vaccinated staff was conducted by using daily health questionnaires, on-call infectious disease unit staff, and post-vaccination web-based questionnaires to identify and test symptomatic HCWs. [21]

According to ICMR Director-General Dr Balram Bhargava the post-vaccination breakthrough infection rate in India for COVID-19 is not more than 0.04 percent. Among 11.6 crore recipients of Covishield, 17,145 (0.02 percent) tested positive after receiving the first dose and 5,014 (0.03 percent) were detected with the coronavirus infection after the second dose.[22] Saad-Roy et al, in their study discussed inevitable uncertainties facing vaccine rollout and they found that onedose strategies generally reduce infections in the short term, while the long-term outcome depends on immune robustness.[23] Yadav PD et al study reported neutralization data in the broader B.1.617 variant category and suggested that convalescent serum samples from persons with Covid-19 and from recipients of the BBV152 vaccine (Covaxin) were able to neutralize variants in the B.1.617 lineage.[24]

In this study $92.48 \% \mathrm{HCWs}$ were vaccinated, 817 (38.1\%) $\mathrm{HCW}$ received both doses. Other HCWs were not vaccinated because of medical conditions, allergies or some misconception about vaccination. The majority $(60.7 \%)$ of infections occurred within first 2 weeks of the vaccination. HCWs testing positive after receiving both doses of vaccinations were 33 while 102 had positive test results after the first dose. Our results were concordant with Keehner J et al and Mary Van Beusekom. [25, 26] In a study of Bouton TC et al, the majority of post vaccination SARS-CoV-2 cases occur in the two weeks following the first dose.[27]

In our study vaccination prevalence was $6.3 \%$ (135/2140). Our results were concordant with the result of Thompson et al study, prevalence was $5.2 \%(205 / 3,950)$ [28] while discordant with study of Bouton et al having prevalence of $1.3 \%$ $(96 / 7,109)$ infected HCWs who received at least one dose, $0.3 \%(17 / 5,913) \mathrm{HCW}$ given both doses.[27] And Amit S et al study showed $0.54 \%$ infection prevalence in vaccinated HCWs.[21] Multiple factors could be responsible for the higher Prevalence of Covid infection in our study compared to other studies. Most of the HCWs in this study were frontline HCWs working in COVID wards. The time period of study of prevalence of infection coincided with the second surge of Pandemic and a smaller number of study population in our study as compared to other studies.

As per WHO there should be a minimum criterion for any acceptable COVID-19 vaccine having clear demonstration of efficacy (on a population basis) ideally with $\sim 50 \%$ point estimate". The efficacy can be assessed against severity of disease, and/or shedding/transmission" endpoints.[29] Covishield has completed its Phase III trials in the UK and the bridging trial in India. However, vaccine effectiveness against the disease and safety in a larger group of people over the duration of 1-2 years is not completed. [11] However our study describes real clinical scenarios during the early period of post vaccination. This is not an efficacy study.

The new delta plus variant cases reported in several states of India including Maharashtra, Kerala and other states.[30] Bernal JL et al found the absolute difference in vaccine effectiveness against symptomatic disease with one dose of vaccine with the delta variant as compared with the alpha variant. However, the differences in vaccine effectiveness after two doses were small.[31] Till now 42,78,82,261 total vaccination doses have been administered and still Indian population needs to be vaccinated totally as early as possible to be prepared for subsequent waves and new variants of Covid 19

In our study most $(87.4 \%)$ of the infected HCWs also had had Mild disease, and only two severe cases (1.5\%), one of them 
died due to infection. According to research study of Jeff Burgess Kris Hanning from University of Arizona Health Sciences, People who contract COVID-19 after vaccination are likely to have a lower viral load, have milder symptoms and experience a shorter infection time than those who are unvaccinated. However, breakthrough infections do occur, the level of infection and impact of the disease are significantly reduced.[32] The current COVID-19 vaccination drive is not really for controlling the spread, but to protect those who are likely to develop severe disease.[33] Our result was concordant with them.

Several new kinds of variants may emerge in the future as it will keep mutating. Large-scale vaccination is the most successful and cost-effective tool for the control or eradication of viral diseases. However vaccinated individuals might get infected and transmit the virus. Hence Infection control practices like masking, social distancing, appropriate PPE and hand sanitization and vaccination are mandatory.

\section{CONCLUSION}

Ensuring the safety of HCWs by priority vaccination is essential to combat the pandemic Breakthrough infections can occur; however, disease severity is milder post vaccination. Early identification of asymptomatic carriers even in vaccinated individuals is important.Post-vaccination surveillance on a large scale is important as it helps to study unexpected adverse risks caused due to the vaccination process. Preparedeness for emerging Covid variants, following Covid-19-appropriate behavior and large scale vaccination is need of time.

\section{REFERENCES}

1. Cheng ZJ, Shan J. 2019 Novel coronavirus: where we are and what we know. Infection. 2020;48:155-63.

2. Rothan HA, Byrareddy SN. The epidemiology and pathogenesis of coronavirus disease (COVID-19) outbreak.J Autoimmun. 2020;102433

3. Sabetian, G., Moghadami, M., HashemizadehFardHaghighi, L. et al. COVID-19 infection among healthcare workers: a cross-sectional study in southwest Iran.Virol J 18,58 (2021)

4. Yadav PD, Nyayanit DA, Sahay RR, et al. Isolation and characterization of the new SARS-CoV-2 variant in travellers from the United Kingdom to India:VUI202012/01 of the B.1.1.7 lineage.JTravel Med 2021;28.

5. Menon GI. The Novel Coronavirus Variants and India's Uncertain Future. The Wire Science. 2021; Available at: https://science.thewire.in/health/sars-cov2-variantsbl17-bl617-india-second-w ave-uncertain-future/. Accessed 22 April 2021.

6. European Centre for Disease Prevention and Control. Threat assessment brief: emergence of SARS-CoV-2 B.1.617 variants in India and situation in the EU/EEA. May 11,2021

7. Li Q,Wu J, Nie J, et al. The impact of mutations in SARS-CoV-2 spike on viral infectivity and antigenicity. Cell 2020;182(5):1284.e9-1294.e9.

8. Johnson BA, Xie X, Kalveram B, et al. Furin cleavage site is key to SARS-CoV-2 pathogenesis. August 26,2020

9. There's now a delta plus variant of covid -19- what does this mean? Gavi,The Vaccine Alliance. 29 June 2021 - by Priya Joi.

10. Dooling K, McClung N, Chamberland M, Marin M,Wallace M, Bell BP et al. The Advisory Committee on Immunization Practices' Interim Recommendation for Allocating Initial Supplies of COVID-19 Vaccine - United States, 2020. MMWR Morb MortalWkly Rep.2020 Dec 1 1;69(49):1857-1859.

11. FAQs on COVID 19 vaccine for Healthcare providers and ...https://www. mohfw.gov.in > FAQsforHCWs\&FLWs.

12. Moderna's Covid vaccine approved for use in India. Rhythma Kaul, Hindustan Times, New Delhi.JUN 30,2021.

13. Rubin EJ, Baden LR, Udwadia ZF, Morrissey S. India's Covid-19 crisis. N Engl J Med2021;384(18):e84-e84.

14. Sampath Kumar NS, Chintagunta AD, Jeevan Kumar SP, Roy S, Kumar M. Immunotherapeutics for Covid-19 and post vaccination surveillance. 3 Biotech.2020 Dec;10(12):527.

15. The effects of virus variants on COVID-19 vaccines. World Health Organisation. 1 March 2021.

16. Bleier BS, Ramanathan M Jr, Lane AP. COVID-19 vaccines may not prevent nasal SARS-CoV-2 infection and asymptomatic transmission. Otolaryngol Head Neck Surg. 2021; 164(2):305-307.

17. Zhaori G, Sun M, Ogra PL. Characteristics of the immune response to poliovirus virion polypeptides after immunization with live or inactivated polio vaccines. Infect Dis. 1988; 158(1): 160- 165

18. Moderna's Covid vaccine becomes 4 th jab to get emergency use approval in India: Govt. Aparna Banerjea. LiveMint India.JUN 29, 2021.

19. Corman VM, Landt O, Kaiser M, Molenkamp R, Meijer A, Chu DK et al. Detection of 2019 novel coronavirus (2019-nCoV) by real-time RT-PCR. Euro Surveill.2020 Jan;25(3):2000045.

20. Schiavone, M, Gasperetti, A, Mitacchione, G, Viecca, M, Battista Forleo, G. Response to: COVID-19 re-infection. Vaccinated individuals as a potential source of transmission. Eur J Clin Invest. 2021;51:e13544.

21. Amit S, Beni SA, Biber A, Grinberg A, Leshem E, Regev-Yochay G. Postvaccination COVID-19 among Healthcare Workers, Israel. Emerg Infect Dis. 2021 Apr;27(4):1220-1222.
22. Post-vaccination breakthrough infection rate in India not more than $0.04 \%$ : ICMR MONEYCONTROL NEWS, APRIL 21,2021 / 10:30 PM IST

23. Saad-Roy CM, Morris SE, Metcalf CJE, et al.Epidemiological and evolutionary considerations of SARS-CoV-2 vaccine dosing regimes. Science. 2021;372(6540):363-370.

24. Yadav PD, Sapkal GN, Abraham P, et al. Neutralization of variant under investigation B. 1.617 with sera of BBV152 vaccines. April 23,2021

25. Keehner J, Horton LE, Pfeffer MA, et al. SARS-CoV-2 Infection after Vaccination in Health Care Workers in California. N Engl JMed. 2021.

26. Mary Van Beusekom. Risk of COVID very low in vaccinated medical workers: study. CIDRAP News. March 24,2021.

27. Bouton TC, Lodi S, Turcinovic J, Weber SE, Quinn E. COVID-19 vaccine impact on rates of SARS-CoV-2 cases and post vaccination strain sequences among healthcare workers at an urban academic medical center: a prospective cohort study. medRxiv [Preprint]. 2021 Mar 31:2021.03.30.21254655.

28. Thompson MG, Burgess JL, Naleway AL, et al. Interim Estimates of Vaccine Effectiveness of BNT162b2 and mRNA-1273 COVID-19 Vaccines in Preventing SARS-CoV-2 Infection Among Health Care Personnel, First Responders, and Other Essential and Frontline Workers - Eight U.S. Locations, December 2020-March 2021. MMWR Morb Mortal Wkly Rep. 2021; 70(13):495-500

29. Susanne H Hodgson, Kushal Mansatta, Garry Mallett, Victoria Harris, Katherine R W Emary, Andrew J Pollard. What defines an efficacious COVID19 vaccine? A review of the challenges assessing the clinical efficacy of vaccines against SARS-CoV-2. ww.thelancet.com/infection Vol 21 February 2021.

30. Delta Plus: List of states where this new Covid variant has been reported so far.Poulomi Ghosh. hindustantimes.com. JUN 24,2021.

31. Bernal JL, Andrews N, Gower C, Gallagher E, Simmons R, Thelwall S, et al. Effectiveness of Covid-19 Vaccines against the B.1.617.2 (Delta) Variant. N EnglJMed.July 21,2021.DOI 10.1056/NEJMoa2108891.

32. Jeff BurgessKris Hanning. COVID-19 Vaccine Reduces Severity, Length, Viral Load for Those Who Still Get Infected. University of Arizona Health Sciences. June 30,2021.

33. Bindu Shajan Perappadan. Coronavirus / Vaccines appear effective in preventing severe illnesses caused by COVID-19 variants of concern in India, says JIPMER Director.The Hindu.com. MARCH 25,2021. 